\title{
On the Most General Form of the Compatibility Equations and the Conditions of Integrability of Strain Rate and Strain
}

\author{
Edmund H. Brown
}

\begin{abstract}
The most general forms of the equations of compatibility are derived by a very simple analysis in terms of the material strain rate tensor and the rate of change of the RiemannChristoffel curvature tensor during the deformations. It is shown that recent statements on the significance of the compatibility equations, essentially that their satisfaction is equivalent to the condition that the space be locally Euclidean, are misinterpretations based on restricted forms of the equations. In addition, a new form of the conditions of integrability of strain is derived in terms of the rate of change of the curvature tensor.
\end{abstract}

\section{Definitions and Notations}

Because of the great variety of usage of authors in the field, a few of the definitions and notations employed here will be discussed briefly. However, a general knowledge of tensor analysis and modern elasticity is assumed. For the most general, and at the same time most readable introduction to tensor analysis, including applications to elasticity, the reader is referred to Brillouin [1]; ${ }^{1}$ for an excellent and thorough review of modern elasticity througin 1953, despite a frequently cumbersome notation, the paper of Truesdell [2] is highly recommended; for more recent general discussions, the treatise of Green and Zerna [3], or the article of Doyle and Ericksen [4] should be consulted.

To introduce the strain tensor, we imagine a complete $n$-dimensional material continuum $G$ which, with an arbitrarily chosen coordinate system $x^{i}$, is defined by the form of the metric tensor $g_{i j}=g_{i j}\left(x^{k}\right)$. To show the covariant character of some of the quantities involved it will occasionally be necessary to transform from one coordinate system to another. Following Craig [5] we shall indicate quantities in one coordinate system by use of indices from the group $i, j, k, h, l$, and quantities in a second coordinate system by indices from the group $p, q, r, s, t$. Thus, such a transformation will be defined by an analytic relation of the form $x^{r}=x^{r}\left(x^{k}\right)$. Further, for convenience, the components of the transformation matrices $\partial x^{k} / \partial x^{r}$ or $\partial x^{r} / \partial x^{k}$ will be denoted by $X_{r}^{k}=\partial x^{k} / \partial x^{r}$ or $X_{k}^{r}=\partial x^{r} / \partial x^{k}$. Similarly, $\partial^{2} x^{k} / \partial x^{s} \partial x^{r}$ will be denoted by $X_{r s}^{k}$. During such a coordinate transformation, then, in one physical state, the components of the metric tensor will transform according to the tensor law, $g_{r s}=g_{i j} X_{r}^{i} X_{s}^{i}$, the Christoffel symbols, or connections, will transform according to the Christoffel law, $\Gamma_{. j k}^{i}=\Gamma_{. s t}^{r} X_{j}^{s} X_{k}^{t} X_{r}^{i}+$ $X_{j k}^{s} X_{s}^{i}$, and the components of the Riemann-Christoffel curvature tensor by the tensor law, $R_{p q \mid r s}=R_{i j \mid k h} X_{p}^{i} X_{q}^{j} X_{r}^{k} X_{s}^{h}$. Note that we use a short vertical stroke between indices to emphasize various symmetry properties. In addition, following Green and Zerna, we use a full vertical stroke behind a quantity, as in the identity $\left.g_{i j}\right|_{k} \equiv 0$, to indicate the covariant derivative.

A material coordinate system is defined by assigning a value $x^{i}$ to each particle of the material continuum, which it retains during any subsequent deformation. We shall assume that the deformation, itself, depends on a single scalar parameter $\mu$, independent of the coordinates $x^{k}$, which in dynamic problems can be identified with the time $t$. Since the material coordinates of a particle do not change during a deformation, we must have $x^{k}\left(\mu_{2}\right)=x^{k}\left(\mu_{1}\right)$. However, during the progress of the deformation the intrinsic quantities associated with the space, such as the metric tensor $g_{i j}$, must change, assuming a continuous set of values $g_{i j}\left(\mu_{1}\right), g_{i j}\left(\mu_{2}\right), \ldots$. When we wish to indicate several stages during a deformation, the appropriate values of $\mu_{1}, \mu_{2}, \ldots$. will be used, but more often only two stages, an "initial" state and a "final" state, are of interest, so that, for instance, we can write for the "initial" metric tensor, $g_{i j}$, and for the "final" metric tensor $\bar{g}_{i j}$. In such cases, we shall make a general practice of indicating a final state by placing bars over quantities which in the initial state are unbarred.

1 Figures in brackets indicate the literature references at the end of this paper. 
At this time, it is well to note that, parallel to the "material" representation of quantities, a "spatial" treatment can be used: A complete $n$-dimensional geometric, or spatial, continuum $H$ can be specified, using an arbitrarily chosen fixed spatial coordinate system $y^{i}$, by the form of the spatial metric tensor, $h_{i j}=h_{i j}\left(y^{k}\right)$. We imagine that the deformation of $G$ takes place in $H$, that is, that there is a one-to-one correspondence between the "particles" of $G$ and the "points" of $H$ which, however, varies in form with $\mu$. Thus, there is a "mapping" of $G$ on $H$ by $y^{i}=y^{i}\left(x^{k}, \mu\right)$, whose variation with $\mu$ represents the deformation of the material continuum. Since during the deformation, that is, during the variation of $\mu$, the particles of $G$ move through the points of $H, y^{i}\left(x^{k}, \mu_{2}\right) \neq y^{i}\left(x^{k} \mu_{1}\right)$, but at each given point $y^{i}$, spatial quantities such as $h_{i j}$ and the spatial Riemann-Christoffel curvature tensor $S_{i \jmath \mid k h}$ remain constant. There is nothing of course to prevent one assuming that the space $H$ may change with parameters other than $\mu$, a device which could possibly be of some use in discussions of plasticity; but for simplicity we shall avoid this here. In any case, a necessary condition that $G$ remain in $H$ is

at each value of $\mu$.

$$
R_{i j \mid k h}=S_{p q \mid r s} \frac{\partial y^{p}}{\partial x^{i}} \frac{\partial y^{q}}{\partial x^{j}} \frac{\partial y^{r}}{\partial x^{k}} \frac{\partial y^{s}}{\partial x^{h}}
$$

Quantities such as strain can be related to either the material or spatial systems, whereas the displacement $u^{i}$ is essentially spatial. Since we intend to discuss strain only, for convenience we will use chiefly a material representation and make little further reference to the spatial treatment. We should remark, however, that one of the principal applications of the results for non-Euclidean continua is in cases where (unlike our definitions above) the dimension of $G$ is $m$, the dimension of $H$ is $n$, and $m<n$, since they are useful in reduced problems involving shells and curved rods. These problems, however, require the introduction of $n-m$ other $m$ th-order quantities ("changes in curvature", "changes in torsion", etc.) in addition to the strain, so that we shall not consider such possibilities here.

\section{Introduction of Strain and Derivation of Results}

We shall take as a measure of finite strain the quantity

$$
{ }^{(12)} e_{i j}\left(x^{k}\right)=\frac{1}{2}\left[g_{i j}\left(x^{k}, \mu_{2}\right)-g_{i j}\left(x^{k}, \mu_{1}\right)\right],
$$

or, if we wish to think only of some particular initial and final states,

$$
e_{i j}\left(x^{k}\right)=\frac{1}{2}\left[\bar{g}_{i j}\left(x^{k}\right)-g_{i j}\left(x^{k}\right)\right],
$$

since-even though it is less used in the modern theory of finite elasticity-it reduces to the familiar strain tensor of "classical" theory, such as is found in Love [6], in the infinitesimal case. According to the above definition, strain depends merely on the initial and final states, and is independent of the way in which the deformation took place. Such a definition is too general for strain to be uniquely related to stress in more complex cases involving permanent set or plastic behavior. Thus, we are led to the introduction of the rate of strain tensor $\dot{e}_{i j}$ defined by

$$
\dot{e}_{i j}=\frac{\partial e_{i j}}{\partial \mu}=\frac{1}{2} \frac{\partial g_{i j}}{\partial \mu} .
$$

We note that, since differentiation with respect to the scalar parameter $\mu$ does not affect the character of $g_{i j}, \dot{e}_{i j}$ like $g_{i j}$ is a tensor in the $x^{k}$ coordinate system for a given value of $\mu$. Similarly, since both $\bar{g}_{i j}$ and $g_{i j}$ are tensors, the finite strain ${ }^{(12)} e_{i j}$ is a tensor. However, though $\bar{x}^{k}=x^{k}$ for all $\mu$, the identities (based on the hypothesis of a Riemann space, that is, that a unique measure exists) $\bar{g}_{i j} \mid \bar{k} \equiv 0$ and $\left.g_{i j}\right|_{k} \equiv 0$ do not allow us to conclude either that $g_{i j} \mid \bar{k} \equiv 0$, or $\left.\bar{g}_{i j}\right|_{k} \equiv 0$, since $g_{i j}$ is not the metric tensor of $\bar{G}$, nor $\bar{g}_{i j}$ the metric tensor of $G$. In $G, \bar{g}_{i j}$ is merely a tensor field and not the metric tensor; similar statements can be made about the character of $g_{i j}$ in $\bar{G}$. 
Now, considering $\dot{e}_{i j}$ to be a tensor field in $G$, in accord with the usual requirements for raising and lowering indices, we must have

and

$$
\dot{e}^{i j}=g^{i k} g^{j h} \dot{e}_{k h}=-\frac{1}{2} \frac{\partial g^{i j}}{\partial \mu}
$$

$$
\dot{e}_{. j}^{i}=g^{i k} \dot{e}_{k j}=\frac{1}{4}\left(g^{i k} \frac{\partial g_{k j}}{\partial \mu}-g_{k j} \frac{\partial g^{i k}}{\partial \mu}\right) .
$$

Just as the metric tensor $g_{i j}$ changes during the deformation, the Christoffel connection $\Gamma_{. j k}^{i}$ based on $g_{i j}$ must change. We shall define the rate of change $\dot{E}_{. j k}^{i}$ of $\Gamma_{. j k}^{i}$ by

$$
\dot{E}_{\cdot j k}^{i}=\frac{\partial \Gamma_{. j k}^{i}}{\partial \mu} .
$$

However, since $\Gamma_{. j k}^{i}$ transforms, when the coordinates are changed at one physical state, by the Christoffel law, and none of the quantities $x^{k}, X_{r}^{k}, X_{r s}^{k}$ are functions of $\mu$,

$$
\dot{E}_{. s t}^{r}=\frac{\partial \Gamma_{. s t}^{r}}{\partial \mu}=\frac{\partial \Gamma_{. j k}^{i}}{\partial \mu} X_{s}^{j} X_{t}^{k} X_{i}^{r}=\dot{E}_{. j k}^{i} X_{s}^{i} X_{t}^{k} X_{i}^{r}
$$

which shows that-unlike $\Gamma_{. j k}^{i}$ - the quantities $\dot{E}_{. j k}^{i}$, and thus $\dot{E}_{i \mid j k}=g_{i h} \dot{E}_{. j k}^{h}$, are tensors, a fact first pointed out by Bergmann [7].

Just as $\Gamma_{. j k}^{i}$ can be expressed, for a Riemann space, in terms of the metric tensor,

$$
\Gamma_{. j k}^{i}=\frac{1}{2} g^{i h}\left[\frac{\partial g_{h j}}{\partial x^{k}}+\frac{\partial g_{h k}}{\partial x^{j}}-\frac{\partial g_{j k}}{\partial x^{h}}\right],
$$

so the $\dot{E}_{. j k}^{i}$ can be written in terms of the strain rate $\dot{e}_{i j}$; for

$$
\dot{E}_{. j k}^{i}=\frac{\partial \Gamma_{. j k}^{i}}{\partial \mu}=\frac{1}{2} \frac{\partial g^{i h}}{\partial \mu}\left[\frac{\partial g_{h j}}{\partial x^{k}}+\frac{\partial g_{h k}}{\partial x^{j}}-\frac{\partial g_{j k}}{\partial x^{h}}\right]+\frac{1}{2} g^{i h} \frac{\partial}{\partial \mu}\left[\frac{\partial g_{h j}}{\partial x^{k}}+\frac{\partial g_{h k}}{\partial x^{j}}-\frac{\partial g_{j k}}{\partial x^{h}}\right]
$$

or, since $g_{i j}$ is an analytic function of $x^{i}$ and $\mu$, and $x^{i}$ and $\mu$ are independent,

$$
\dot{E}_{. j k}^{i}=-2 \dot{e}^{i h} \Gamma_{h \mid j k}+g^{i h}\left[\frac{\partial \dot{e}_{h j}}{\partial x^{k}}+\frac{\partial \dot{e}_{h k}}{\partial x^{j}}-\frac{\partial \dot{e}_{j k}}{\partial x^{h}}\right]
$$

Similarly, $\dot{E}_{i \mid j k}$ can be shown to be given by

$$
\dot{E}_{i \mid j k}=g_{i h} \dot{E}_{. j k}^{h}=-2 \dot{e}_{i h} \Gamma_{. j k}^{h}+\left[\frac{\partial \dot{e}_{\imath j}}{\partial x^{h}}+\frac{\partial \dot{e}_{k i}}{\partial x^{j}}-\frac{\partial \dot{e}_{j k}}{\partial x^{i}}\right],
$$

or

$$
\dot{E}_{i \mid j k}=\frac{\partial \Gamma_{i \mid j k}}{\partial \mu}-2 \dot{e}_{i h} \Gamma_{. j k}^{h}
$$

Using the expression for the covariant derivative of the second order tensor $\dot{e}_{i j}$, the three partial derivatives of $\dot{e}_{i j}$ appearing above may be written

$$
\begin{gathered}
\frac{\partial \dot{e}_{h j}}{\partial x^{k}}=\left.\dot{e}_{h j}\right|_{k}+\dot{e}_{l j} \Gamma_{. h k}^{l}+\dot{e}_{h l} \Gamma_{. k j}^{l} \\
\frac{\partial \dot{e}_{h k}}{\partial x^{j}}=\left.\dot{e}_{h k}\right|_{j}+\dot{e}_{l k} \Gamma_{. h j}^{l}+\dot{e}_{h l} \Gamma_{. k j}^{l} \\
-\frac{\partial \dot{e}_{j k}}{\partial x^{h}}=-\left.\dot{e}_{j k}\right|_{h}-\dot{e}_{l k} \Gamma_{. j h}^{l}-\dot{e}_{j l} \Gamma_{. k h}^{l} .
\end{gathered}
$$


On adding these expressions, interchanging indices where symmetry permits, we obtain

$$
\frac{\partial \dot{e}_{h j}}{\partial x^{k}}+\frac{\partial \dot{e}_{h k}}{\partial x^{j}}-\frac{\partial \dot{e}_{j k}}{\partial x^{h}}=\left.\dot{e}_{h j}\right|_{k}+\left.\dot{e}_{h k}\right|_{j}-\left.\dot{e}_{j k}\right|_{h}+2 \dot{e}_{h l} \Gamma_{. k j}^{l} .
$$

Introducing this in the expressions for $\dot{E}_{. j k}^{i}$ and $\dot{E}_{i \mid j k}$ in terms of the strain rate, and making use of the fact that $\left.g^{i n}\right|_{k} \equiv 0$ results in the more elegant forms,

and

$$
\dot{E}_{i\lceil j k}=\left.\dot{e}_{i j}\right|_{k}+\left.\dot{e}_{k i}\right|_{j}-\left.\dot{e}_{j k}\right|_{i}
$$

$$
\dot{E}_{. j k}^{i}=\left.\dot{e}_{. j}^{i}\right|_{k}+\left.\dot{e}_{\dot{k}}^{i}\right|_{j}-\left.\dot{e}_{j k}\right|^{i}
$$

We must, finally, consider how the Riemann-Christoffel curvature tensor $R_{\cdot j \mid k n}^{i}$ based on $g_{i j}$ varies during the deformation. Defining $\dot{\mathscr{E}}_{\cdot{ }_{j} \mid k h}=\partial R_{\cdot j \mid k h}^{i} / \partial \mu$, this rate of change of curvature is given by

$$
\begin{aligned}
\dot{\mathscr{E}}_{., j \mid k h} & =\frac{\partial}{\partial x^{k}}\left(\frac{\partial \Gamma_{. j h}^{i}}{\partial \mu}\right)-\frac{\partial}{\partial x^{h}}\left(\frac{\partial \Gamma_{. j k}^{i}}{\partial \mu}\right)+\frac{\partial \Gamma_{. j h}^{l}}{\partial \mu} \Gamma_{. l k}^{i}+\Gamma_{. j h}^{l} \frac{\partial \Gamma_{. l k}^{i}}{\partial \mu}-\frac{\partial \Gamma_{. j k}^{l}}{\partial \mu} \Gamma_{. l h}^{i}-\Gamma_{. j k}^{l} \frac{\partial \Gamma_{. l h}^{i}}{\partial \mu} \\
& =\frac{\partial \dot{E}_{. j h}^{i}}{\partial x^{k}}+\dot{E}_{. j h}^{l} \Gamma_{. l k}^{i}-\dot{E}_{. l h}^{i} \Gamma_{. j k}^{l}-\dot{E}_{. l \jmath}^{i} \Gamma_{. h k}^{l}-\left(\frac{\partial \dot{E}_{. j k}^{i}}{\partial x^{h}}+\dot{E}_{. j k}^{l} \Gamma_{. l h}^{i}-\dot{E}_{. l k}^{i} \Gamma_{. j h}^{l}-\dot{E}_{. . j l}^{i} \Gamma_{. k h}^{l}\right),
\end{aligned}
$$

or

$$
\dot{\mathscr{E}}_{. j \mid k h}=\dot{E}_{\cdot j h \mid k}^{i}-\left.\dot{E}_{. j k}^{i}\right|_{h}
$$

and similarly,

$$
\dot{\mathscr{E}}_{i j \mid k h}=g_{i l} \dot{\mathscr{E}}_{\cdot j \mid k h}=\frac{\partial R_{i j \mid k h}}{\partial \mu}-2 \dot{e}_{i l} R_{\cdot j \mid k h}^{l}=\left.\dot{E}_{i|j h|}\right|_{k}-\left.\dot{E}_{i \mid j k}\right|_{h}
$$

both of which are especially elegant forms for the rate of change of the curvature tensor.

Just as in the case of $\dot{E}_{i \mid j k}$, we can express $\dot{\mathscr{E}}_{i j \mid k h}$ in terms of the strain rate, and, in doing so, we obtain the equations of compatibility in their most general form. By substitution and simple algebra these identities may be written

$$
\dot{\mathscr{E}}_{i j \mid k h}=\left(\left.\dot{e}_{i j}\right|_{h k}-\left.\dot{e}_{i j}\right|_{k h}\right)+\left(\left.\dot{e}_{i h}\right|_{j k}-\left.\dot{e}_{i k}\right|_{j h}\right)-\left(\left.\dot{e}_{j h}\right|_{i k}-\left.\dot{e}_{j k}\right|_{i h}\right)
$$

It is well to examine the significance of these equations. There is no reason for us to assume that the strain rate is a tensor function (which would require that $\dot{e}_{i j}$ satisfy certain conditions of integrability), for in the most general case, the $\dot{e}_{i j}$ would be merely the components of a tensor field. However, the curvature tensor $R_{i j \mid k n}$ is an intrinsic property of the material continuum $G$ and must be related, as stated previously, to the curvature tensor $S_{i j \mid k n}$ of the spatial continuum $H$, in order that $G$ remain in $H$. Thus, $\dot{\mathscr{E}}_{i j \mid k h}$ must be related to $S_{i j \mid k n}$ by an equation of the form

$$
\dot{\mathscr{E}}_{\cdot j \mid k h}=S_{\cdot q \mid r s}^{p} \frac{\partial}{\partial \mu}\left[\frac{\partial x^{i}}{\partial y^{p}} \frac{\partial y^{q}}{\partial x^{i}} \frac{\partial y^{r}}{\partial x^{k}} \frac{\partial y^{s}}{\partial x^{h}}\right]
$$

We have assumed that $H$ does not vary, that is, that the value of $S_{i j \mid k h}$ at each point $y^{k}$ is constant. In particular, if $S_{i j \mid k h} \equiv 0, R_{i j \mid k h} \equiv 0$, and thus, of course $\dot{\mathscr{E}}_{i j \mid k h}$ must also vanish. If, however, $S_{i j \mid k h}$ is not zero, $\dot{\mathscr{E}}_{i j \mid k h}$ will be constant, or more strongly, zero, only if other special conditions hold.

The conditions of integrability of the rate of strain tensor must now be investigated. Using the relation for $\dot{\mathscr{E}}_{i j \mid k h}$, and the fact that $R_{i j \mid k h}$ is skew-symmetric in the indices of both pairs, we can write

$$
\dot{\mathscr{E}}_{i j \mid k h}+\dot{\mathscr{E}}_{j i \mid k h}=-2\left[\dot{e}_{i l} R_{\cdot j \mid k h}^{l}+\dot{e}_{j l} R_{\cdot}^{l}{ }_{\cdot i \mid k h}\right] .
$$


However, the equations

$$
\dot{e}_{i l} R_{. j \mid k h}^{l}+\dot{e}_{j l} R_{. i \mid k h}^{l}=\left.\dot{e}_{i j}\right|_{k h}-\dot{e}_{i j \mid h k}
$$

are merely the conditions of integrability; the vanishing of either side of the above equation implies that if $\dot{e}_{i j}$ is subjected to parallel transport around a closed curve, it will return to its initial value at the starting point, and thus, may be considered a tensor function of the coordinates $x^{i}$ rather than merely a tensor field.

Thus, the rate of strain is integrable if any one of the following equations holds identically:

$$
\begin{array}{r}
\dot{e}_{i j \mid k h}-\dot{e}_{i j \mid h k}=0 \\
\dot{e}_{i l} R_{\cdot j \mid k h}^{l}+\dot{e}_{j l} R_{\cdot i \mid k h}^{l}=0 \\
\mathscr{E}_{i j \mid k h}+\dot{\mathscr{E}}_{j i \mid k h}=0,
\end{array}
$$

the last of the equations being equivalent to the requirement that $\dot{\mathscr{E}}_{i j \mid k h}$ be skew-symmetric in its first two indices (it is already skew-symmetric in the second pair as a consequence of its definition). It should be noticed that these integrability conditions are distinct from the compatibility equations, and in particular, it is not necessary for $\dot{\mathscr{E}}_{i j \mid k n}$ to vanish for $\dot{e}_{i j}$ to be integrable.

If, however, the strain rate is integrable, and we substitute the first of the above conditions in the compatibility equations, they take the simpler form

$$
\dot{E}_{i j \mid k h}=\left(\left.\dot{e}_{i h}\right|_{j k}-\left.\dot{e}_{i k}\right|_{j h}\right)-\left(\left.\dot{e}_{j h}\right|_{i k}-\left.\dot{e}_{j k}\right|_{i h}\right) .
$$

In a great many practical cases, either the space is Euclidean, or some special relation may hold, such that $\dot{\mathscr{E}}_{i j \mid k n}=0$, which reduces the compatibility equations to the form

$$
\left.\dot{e}_{i h}\right|_{j k}-\left.\dot{e}_{i k}\right|_{j h}=\left.\dot{e}_{j h}\right|_{i k}-\left.\dot{e}_{j k}\right|_{i h} .
$$

It is of some interest to see the forms which the last equations take when $n \leq 3$. If $n=1$, all indices must be identical and we have, merely, $0=0$. If $n=2$, only two indices can be distinct, resulting in only one independent equation

$$
\left.\dot{e}_{i i}\right|_{k k}+\left.\dot{e}_{k k}\right|_{i i}=\left.2 \dot{e}_{i k}\right|_{i k}, \quad i \neq k,
$$

indices not summed.

If $n=3$, three indices may be distinct, and two independent sets of, in all, six equations may be derived:

$$
\left.\dot{e}_{i i}\right|_{k k}+\left.\dot{e}_{k k}\right|_{i i}=\left.2 \dot{e}_{i k}\right|_{i k} \quad i \neq k,
$$

indices not summed (3 equations), and

$$
\left.\dot{e}_{i i}\right|_{j k}+\left.\dot{e}_{j k}\right|_{i i}=\left.\dot{e}_{i j}\right|_{i k}+\left.\dot{e}_{i k}\right|_{i j}, \quad i \neq j \neq k,
$$

indices not summed (3 equations).

The last two sets of equations, for $n=3$, are equivalent to those given by A. E. H. Love in Cartesian coordinates. Thus, our completely general form of the compatibility equations reduces to the more well-known form for the appropriate conditions.

We note that, since $\mu$ is a scalar parameter independent of $x^{i}$, derivatives of the infinitesimal strain tensor $d e_{i j}=\dot{e}_{i j} d \mu$ may be written $\left.d e_{i j}\right|_{k}=\left.\dot{e}_{i j} d \mu\right|_{k}=\left.\dot{e}_{i j}\right|_{k} d \mu$. Similarly, derivatives of the finite strain $e_{i j}=\int \dot{e}_{i j} d \mu$ may be written $\left.e_{i j}\right|_{k}=\left.\int \dot{e}_{i j} d \mu\right|_{k}=\left.\int \dot{e}_{i j}\right|_{k} d \mu$. Thus, all the relations and conditions derived above hold equally well for both the infinitesimal and finite strain tensors.

In conclusion, we can state the equations of integrability and the equations of compatibility are two independent sets of conditions. Satisfaction of the former implies that the 
space to which the deforming medium is assigned is Euclidean. Satisfaction of the latter implies the medium remains in that assigned space. The fact that both must hold for the solution of actual problems in elasticity should not lead to considering them as identical, for each has its own physical significance.

\section{References}

[1] L. Brillouin, Les tenseurs en méchanique et en élasticité (Dover Publications, New York, N. Y., 1946).

[2] C. Truesdell, The mechanical foundations of elasticity and fluid dynamics, J. Rational Mech. and Anal. 1, Nos. 1 and 2 (1952).

[3] A. E. Green and W. Zerna, Theoretical elasticity (Oxford Univ. Press, New York, N. Y., 1954).

[4] T. C. Doyle and J. L. Ericksen, Nonlinear elasticity, Advances in Appl. Mech. 4, (1956).

[5] H. V. Craig, Vector and tensor analysis (McGraw Hill Book Co., Inc., New York, N. Y., 1943).

[6] A. E. H. Love, Theory of elasticity (Dover Publications, New York, N. Y., 1944).

[7] P. G. Bergmann, Theory of relativity (Prentice-Hall, Inc., New York, N. Y., 1946).

[8] A. S. Lodge, Compatibility equations for large strains, Quart. J. Mech. and Appl. Math. 4, (1951).

[9] F. D. Murnaghan, Finite deformation of an elastic solid (John Wiley \& Sons, Inc., New York, N. Y., 1951).

Boulder, Colo., July 1, 1957. 